Wahbi JAOUADI ${ }^{1}$

Lamia HAMROUNI ${ }^{1}$

Mohsen HANANA ${ }^{2}$

Kaouther MeCHERGUI ${ }^{1}$

Ghazi GADER ${ }^{3}$

Mohamed Larbi KHouJA ${ }^{1}$

${ }^{1}$ Institut national de recherches

en génie rural, eaux et forêts (Inrgref)

de Tunis

Laboratoire d'écologie et amélioration

sylvo-pastorale

Rue Hédi Karray

BP 10, Ariana, 2080

Tunisie

${ }^{2}$ Centre de biotechnologie

de Borj-Cédria

Laboratoire de physiologie moléculaire des plantes

BP 901, Hammam-Lif, 2050

Tunisie

3 Direction générale des forêts Avenue Charles Nicolle 1082 Tunis-Cité El Mahrajène Tunisie

\section{Dynamique de la régénération
d'Acacia tortilis subsp. raddiana \\ Dynamique de la régénération
d'Acacia tortilis subsp. raddiana dans le parc national
de Bou Hedma en Tunise de Bou Hedma en Tunise}

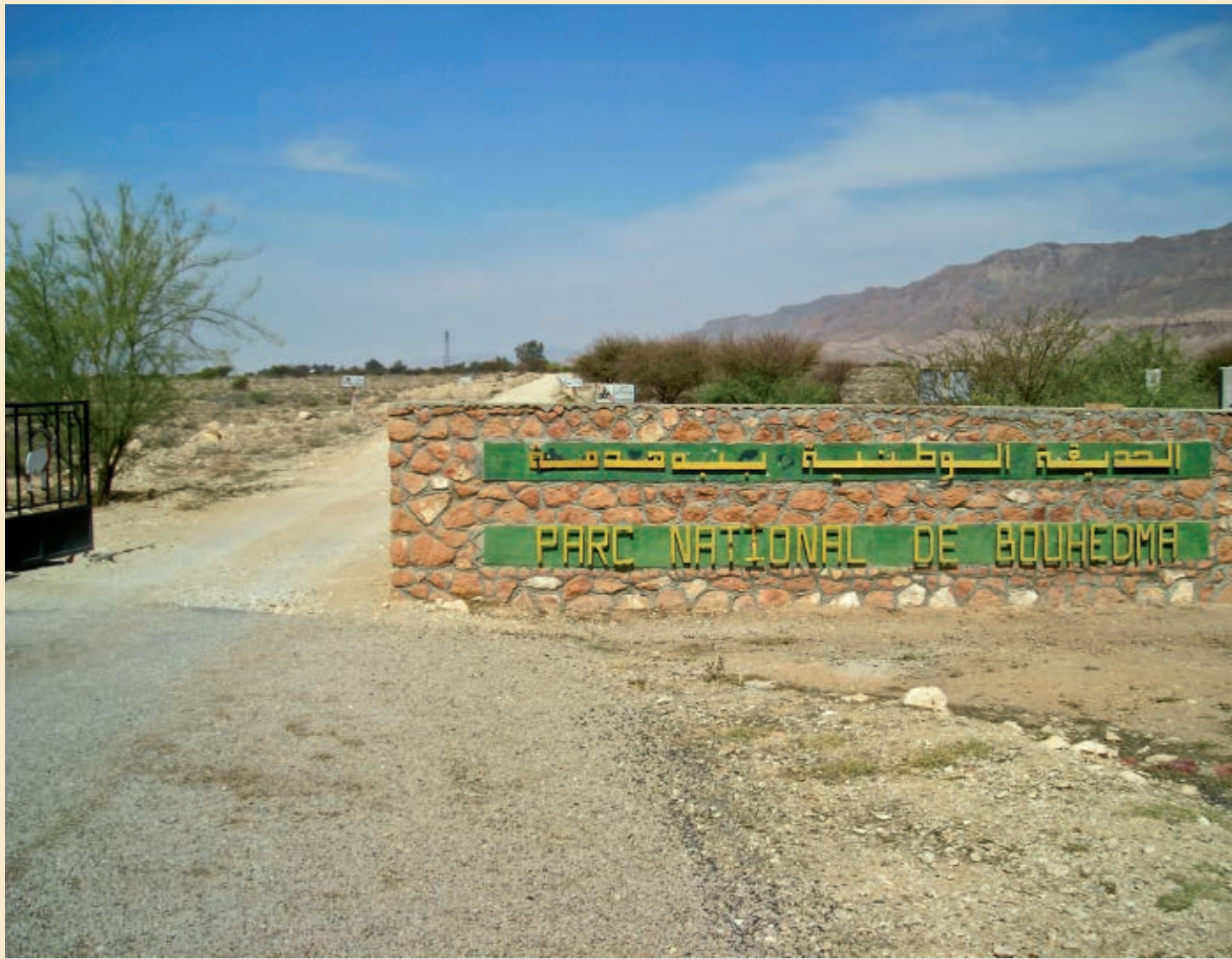

Photo 1.

Entrée du parc national de Bou Hedma (2005).

Photo W. Jaouadi. 


\section{RÉSUMÉ}

\section{DYNAMIQUE DE LA RÉGÉNÉRATION D'ACACIA TORTILIS SUBSP. RADDIANA DANS LE PARC NATIONAL DE BOU HEDMA EN TUNISE}

La présente étude a été réalisée dans le parc national de Bou Hedma, situé dans les zones arides tunisiennes et considéré comme une réserve de la biosphère de l'Unesco dès 1977. Ce parc abrite l'unique steppe arborée à Acacia tortilis (Forsk.) Hayne subsp. raddiana (Savi) Brenan en Tunisie. Notre démarche est basée sur l'utilisation conjointe de l'imagerie satellitaire, des relevés de végétation ainsi que l'observation des caractéristiques dendrométriques de cette espèce. L'interprétation des images satellitaires montre qu'Acacia tortilis occupe une vaste aire de répartition (12\% de la superficie totale du parc) et se localise essentiellement dans les zones mises en défens et sur celles d'épandage qui se caractérisent par un sol squelettique. Cette espèce préfère également les zones dont l'altitude est supérieure à 200 m, sur des sols profonds et à faible pente. Globalement, le recouvrement observé d'Acacia tortilis est inférieur à $10 \%$ en grande partie, avec une présence remarquable de jeunes semis issus de la régénération naturelle et ayant un diamètre supérieur à $10 \mathrm{~cm}$.

Mots-clés : Acacia tortilis, dynamique, régénération, relevés de végétation, zone aride, image satellitaire, Tunisie.

\section{ABSTRACT}

\section{REGENERATION DYNAMICS IN ACACIA TORTILIS SUBSP. RADDIANA IN TUNISIA'S BOU HEDMA NATIONAL PARK}

This study was conducted in Bou Hedma national park, which is in Tunisia's arid zone and was listed as a Unesco Biosphere Reserve in 1977. The park harbours Tunisia's only tree steppe region with Acacia tortilis (Forsk.) Hayne subsp raddiana (Savi) Brenan. Our approach involves a combination of satellite imagery, vegetation surveys and observations of the dendrometric characteristics of this Acacia species. Our interpretation of the satellite images shows that Acacia tortilis occupies a very large area (12\% of the park's total area) and is essentially located in zones with characteristically skeletal soils, as in grazing prohibition zones or detritic-colluvial deposits. The species also prefers zones more than $200 \mathrm{~m}$ above sea level, with deep soils and shallow slopes. Overall, observed Acacia tortilis cover is mostly less than $10 \%$, with a very significant presence of young naturally regenerating seedlings more than $10 \mathrm{~cm}$ in diameter.

Keywords: Acacia tortilis, dynamics, regeneration, vegetation surveys, arid zone, satellite image, Tunisia.

\section{RESUMEN}

\section{DINÁMICA DE LA REGENERACIÓN DE ACACIA TORTILIS SUBSP. RADDIANA, EN EL PARQUE NACIONAL DE BOU HEDMA EN TÚNEZ}

El presente estudio se realizó en el Parque Nacional de Bou Hedma, ubicado en las zonas áridas tunecinas y calificado como reserva de la biosfera por la Unesco desde 1977. Este parque alberga la única estepa arbolada de Acacia tortilis (Forsk.) Hayne subsp. raddiana (Savi) Brenan de Túnez. Nuestro enfoque se basó en el uso conjunto de imágenes satelitales, inventarios de vegetación y la observación de las características dendrométricas de cada especie. La interpretación de las imágenes satelitales muestra que Acacia tortilis ocupa una amplia área de distribución (12\% de la superficie total del parque) que se localiza fundamentalmente en áreas de aprovechamiento prohibido y terrenos aluviales que se caracterizan por su suelo esquelético. Asimismo, esta especie prefiere las zonas situadas por encima de los $200 \mathrm{~m}$, con suelos profundos y pendientes poco pronunciadas. Globalmente, el grado de cobertura observado en Acacia tortilis es inferior al $10 \%$ y, en gran parte, con una notable presencia de plantas jóvenes procedentes de la regeneración natural y con un diámetro superior a $10 \mathrm{~cm}$.

Palabras clave: Acacia tortilis, dinámica, regeneración, inventarios de vegetación, zona árida, imagen satelital, Túnez. 


\section{Introduction}

La zone du Bled Talah, qui fait partie de la Tunisie méridionale (entre les isohyètes 100 et $350 \mathrm{~mm}$ ), connaît depuis la sédentarisation des populations (au début du XXe siècle) de sévères perturbations anthropiques. L'exploitation des ressources offertes par Acacia tortilis (Forsk.) Hayne subsp. raddiana (Savi), Brenan var. raddiana Brenan, depuis le début du XIXe siècle (ZAÂFOURI et al., 1996), conjuguée à la faible résilience de l'écosystème ainsi que la forte pression anthropique de la population, a conduit à une fragilité inéluctable de ce milieu. L'étude est menée au sein du parc national de Bou Hedma qui s'étend sur une superficie de 16448 ha. Il constitue l'unique milieu "refuge » pour les espèces végétales et animales des zones arides tunisiennes menacées d'extinction. Ce travail est mené pour cartographier et caractériser la dynamique de régénération d'Acacia tortilis afin d'évaluer l'état du couvert végétal aussi bien sur le plan quantitatif que qualitatif.

\section{Présentation de la zone d'étude}

Le parc national de Bou Hedma $\left(\mathrm{N}=33^{\circ}\right.$ 30'; $\left.E=9^{\circ} 38^{\prime}\right)$, créé par le décret $n^{\circ}$ 80-1606 du 18 décembre 1980, est localisé en Tunisie centrale (figure 1 et photo 1). Il couvre une superficie de 16448 ha (KAREM, 2001) et comporte trois zones de protection intégrale (respectivement 5114 ha du côté Est autour du Borj Bou Hedma, 2534 ha du côté de l'Oued Haddej au centre du parc, et 1006 ha au Sud-Ouest du parc). Ce parc abrite une faune sauvage diversifiée (photos 2) et une végétation arborée essentiellement représentée par Acacia tortilis (photo 3).

\section{Matériel et méthodes}

Les cartes utilisées sont essentiellement des images du satellite Quick Bird de 0,6 m de résolution spatiale, dont la scène de $5 \mathrm{~km}$ sur $5 \mathrm{~km}$ a été acquise le 9 septembre 2005. D'autres cartes de type topographique à l'échelle de 1/25 000 ont été également utilisées pour la localisation des relevés de végétation. Les logiciels ArcView et Erdas Imagine ont été retenus pour réaliser l'ensemble des traitements d'images et des analyses spatiales nécessaires à l'étude.

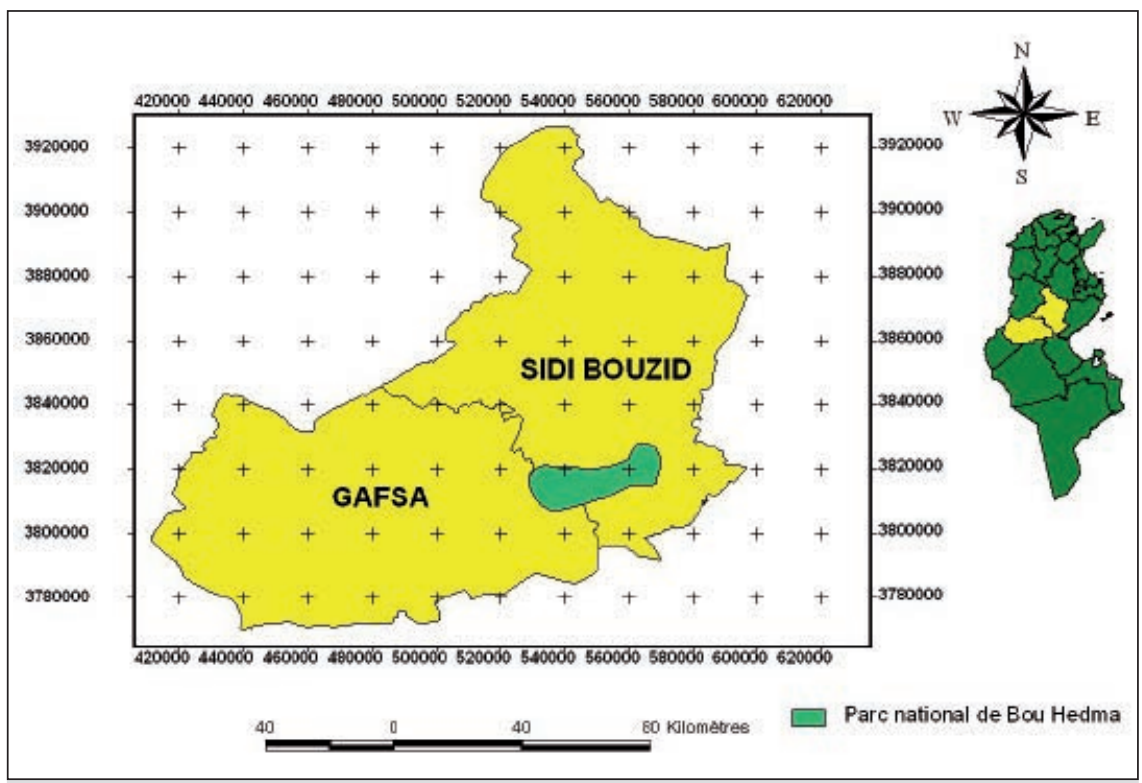

Figure 1.

Localisation du parc national de Bou Hedma.
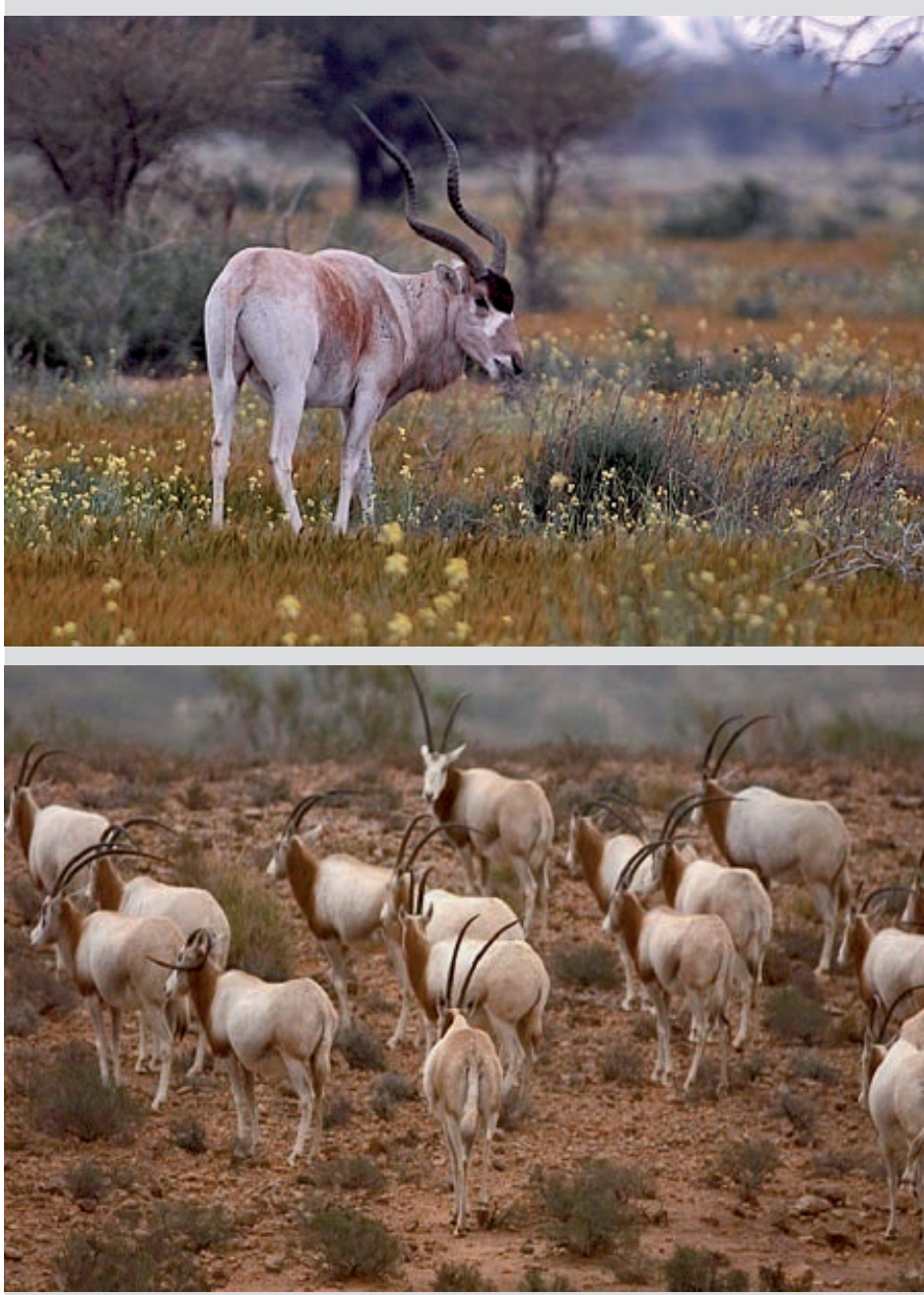

Photos 2.

La faune sauvage du parc national de Bou Hedma en 2005 (antilope : oryx et addax).

Photo W. Jaouadi. 


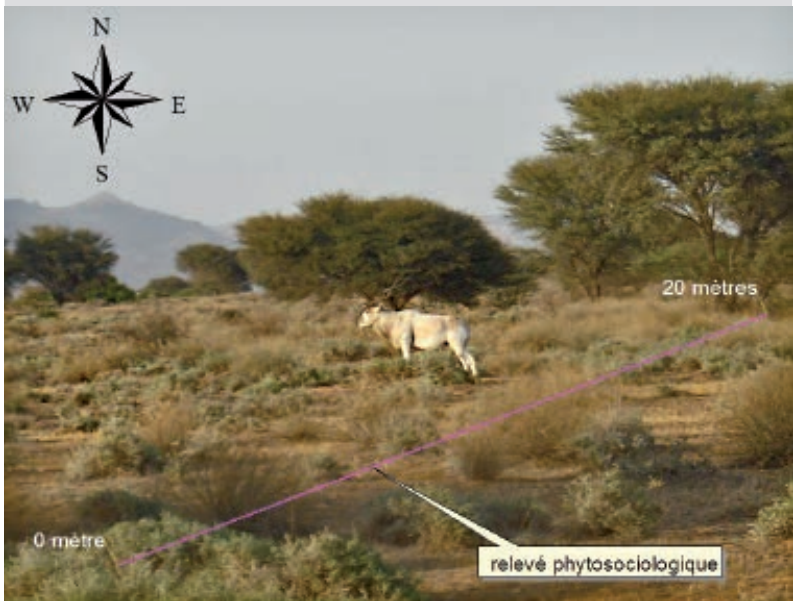

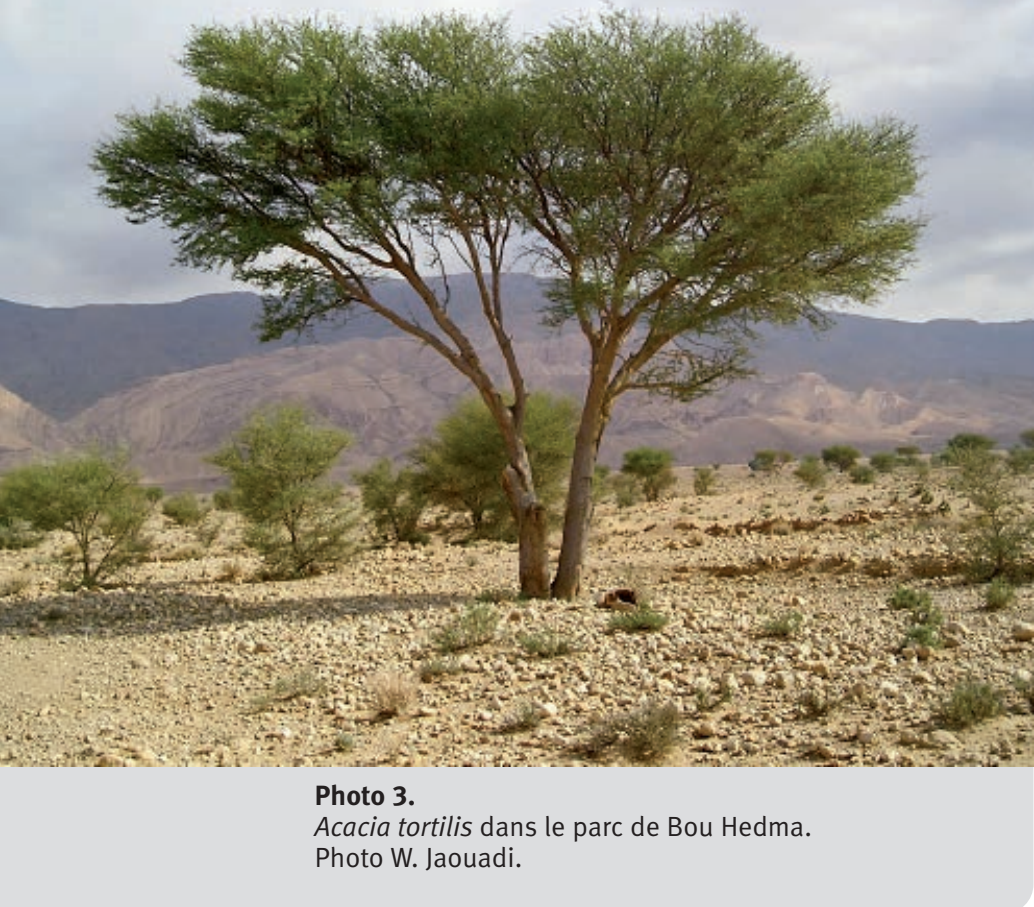

\section{Méthodes}

\section{Mesure du recouvrement}

\section{Sur les images}

Les mesures de recouvrement spatial d'Acacia tortilis ont nécessité le recours à l'analyse numérique des images satellitaires Quick Bird. Ces images ont été préalablement mosaïquées, géoréférencées, géométriquement corrigées et rehaussées pour les rendre exploitables.

Dans un premier temps, une classification non supervisée a été mise en œuvre afin d'obtenir une première localisation de la strate ligneuse de la zone d'étude. Cette approche permet d'avoir une idée non seulement de l'état du peuplement ligneux d'Acacia tortilis, mais aussi de la diversité paysagère du terrain, permettant également de mieux disposer les relevés floristiques sur le terrain. Dans un deuxième temps, une grille de $1 \mathrm{~km}$ de résolution spatiale a été apposée sur les images afin d'élaborer un échantillonnage systématique pour mesurer la densité spatiale des individus d'Acacia tortilis. De là, les classes de densité ont été déterminées, dégageant une stratification basée sur des critères de consistance du peuplement exprimée en pourcentage à l'aide de la grille d'échelle de densité du couvert (Grown Density Scale), variable utilisée du fait de son intérêt écologique et de l'homogénéité de la composition floristique.

Les classes suivantes ont été retenues :

- $20<\mathrm{R}<25 \%$ : peu dense ;

- $15<\mathrm{R}<20 \%$ : moyennement claire ;

- $10<\mathrm{R}<15 \%$ : claire ;

- $5<R<10 \%$ : faible ;

- $\mathrm{R}<5 \%$ : très faible.

\section{Photo 4.}

Transect de végétation.

Photo W. Jaouadi.

Avec $R$ = recouvrement de la strate arborée (somme des surfaces de la projection orthogonale des cimes de l'espèce sur une unité de surface qui est une maille de $1 \mathrm{~km}$ de côté). Ces recouvrements ont été divisés par classe de densité de telle sorte d'avoir un groupement des placettes ayant des recouvrements presque semblables.

\section{Sur le terrain}

Les mesures du recouvrement réalisées sur le terrain ont été plutôt basées sur l'utilisation de la méthode des points quadrats (DAGET, PoIssonet, 1971). Cette méthode de mesure quantitative du recouvrement de la végétation est aisément reproductible d'un endroit à un autre. Son principe de base est d'effectuer des observations à intervalles réguliers le long d'un transect linéaire (photo 4).

Le choix de la station écologique repose sur le critère d'homogénéité de la végétation (composition floristique et conditions écologiques) ainsi que sur les travaux de SCHOENEnBERGER (1986, 1987, 1988, 1991). La longueur du relevé est fixée à $20 \mathrm{~m}$ pour noter toutes les espèces présentes dans la station écologique considérée. Pour chaque relevé, les observations ont été notées chaque $10 \mathrm{~cm}$ pour obtenir 200 points de contact (figure 2). Deux cents relevés de végétation ont été effectués, comprenant chacun les coordonnées géographiques extraites d'une carte topographique à l'échelle de 1/25 000. La composition floristique de chaque relevé est notée sur une fiche technique. Ces relevés floristiques ont été utilisés pour valider l'ensemble des unités homogènes déduites par la classification non supervisée. Cette approche est une étape majeure dans la validation de la répartition spatiale d'Acacia tortilis. Conjointement à la mesure du recouvrement, des mesures dendrométriques ont été également réalisées par la mise en œuvre de neuf placettes d'un hectare chacune (figures 3 et 4). Cinq placettes ont été exécutées au sein d'un peuplement issu d'une régénération naturelle (P1, P2, P3, P4 et $\mathrm{P} 5$ ) et quatre autres au sein d'un peuplement de reboisement (P6, P7, P8 et P9). Ce nombre réduit des placettes est dû à la pauvreté de paysage steppique. Les mesures dendrométriques ont été réalisées sur tous les arbres présents dans toutes les placettes et ont concerné la hauteur, le diamètre basal (10-15 cm du sol) et le diamètre de la couronne des arbres (photo 5). 


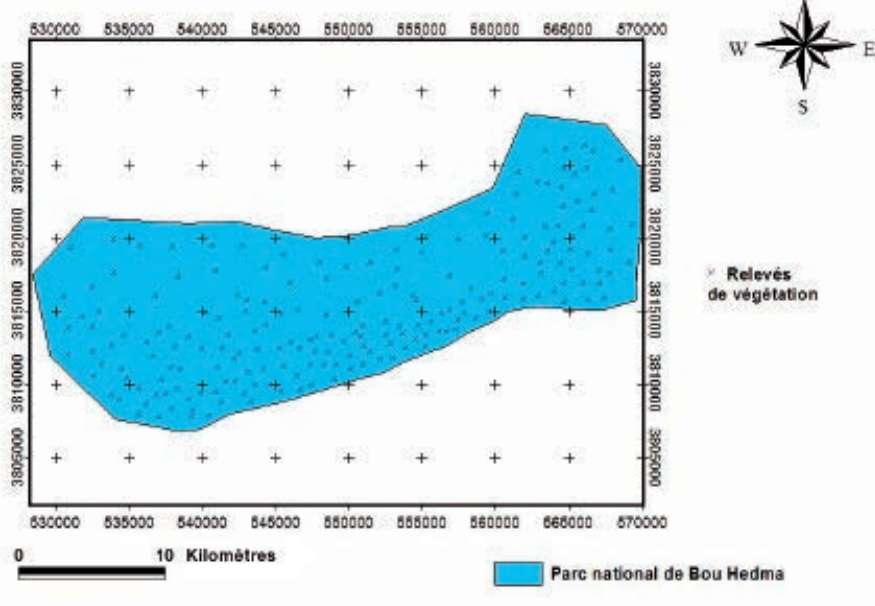

Figure 2.

Localisation des relevés de végétation (200 relevés).

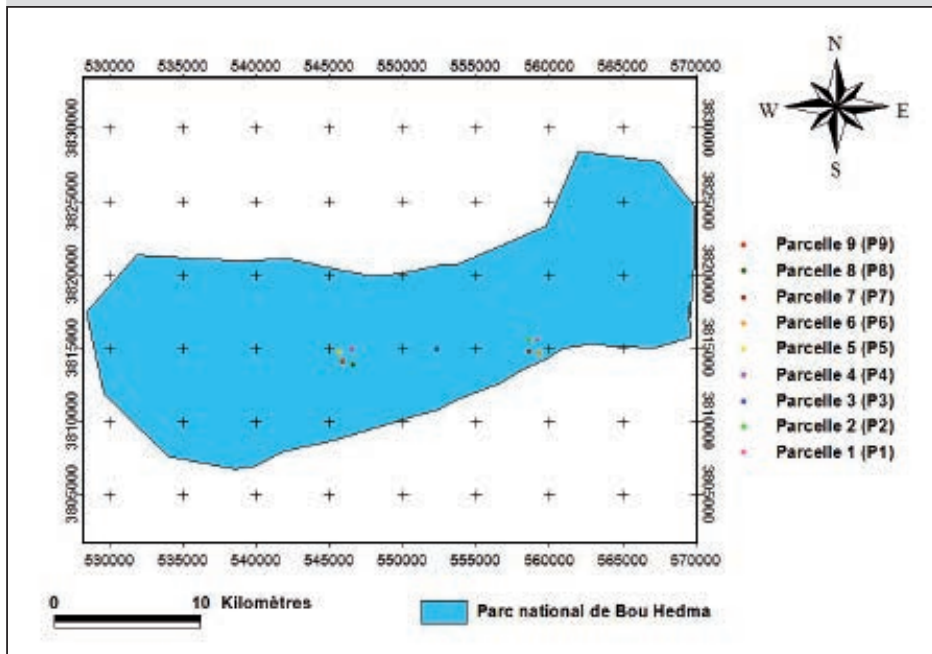

Figure 3.

Localisation des parcelles échantillonnées pour les mesures dendrométriques.

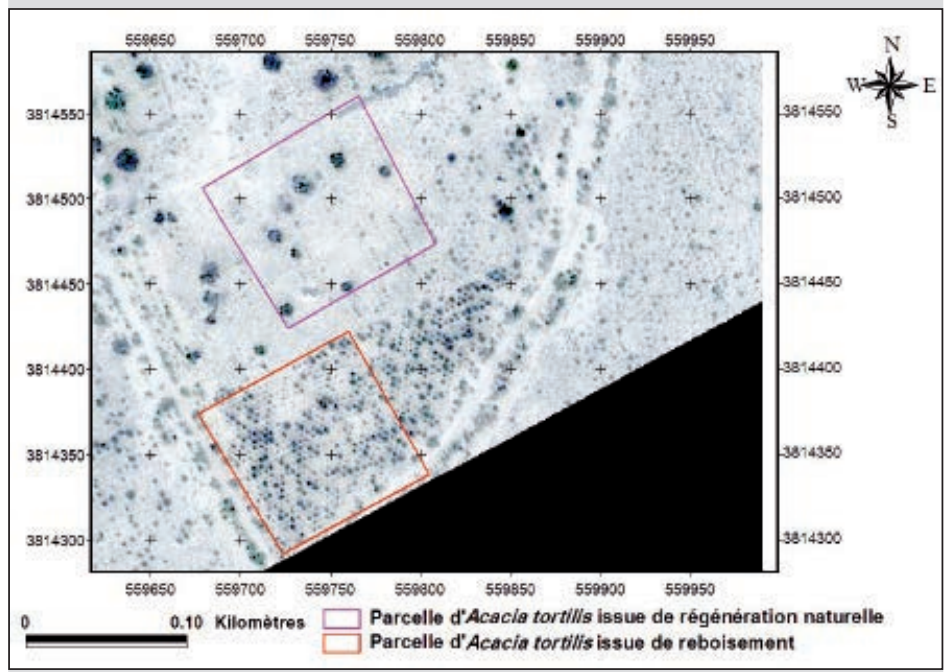

Figure 4.

Parcelles issues de régénération naturelle et de reboisement (septembre 2005).

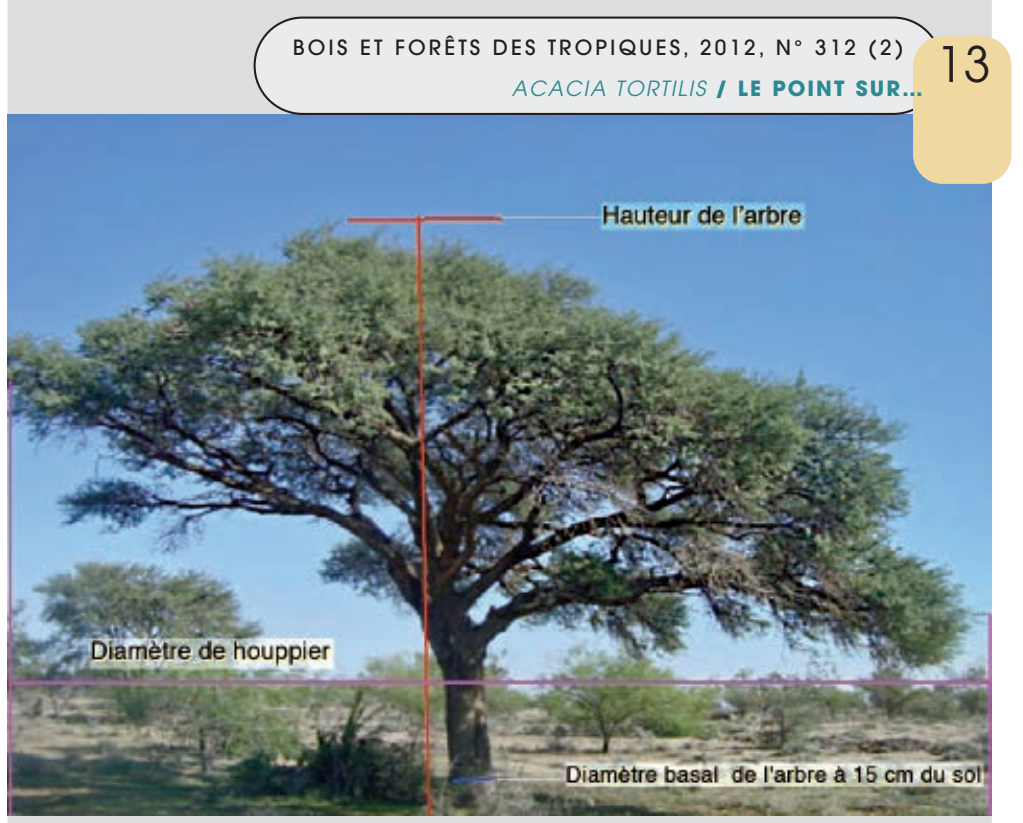

Photo 5.

Dispositif expérimental des mesures dendrométriques.

Photo W. Jaouadi.

\section{Résultats}

L'analyse conjointe des images satellitaires Quick Bird (figure 5) et des observations du recouvrement sur le terrain a permis de cartographier la répartition spatiale d'Acacia tortilis (figure 6). L'analyse de la figure 6 montre que l'aire de distribution d'Acacia tortilis s'étend de Mazouna jusqu'au Orbata. Ce peuplement se localise sur le versant Sud du Jebel Bou Hedma et surtout dans la plaine et les zones d'épandage du Bled Talh (figure 7). Cependant, sur le versant Nord du Jebel Bou Hedma, Acacia tortilis est totalement absent. Les reboisements (figure 8) sont réalisés sur de petites superficies dans les zones de protection intégrale ( 1 : autour du Borj Bou Hedma et II : parc Haddej). L'analyse des relevés de végétation sur l'ensemble de la zone étudiée montre que le paysage du parc national de Bou Hedma est très diversifié. Plusieurs unités de végétation ont été identifiées :

- ligneux bas dominés par Juniperus phoenicea et Olea europea ;

- ligneux bas dominés par Hamada schmittia et Atractylis serratuloides ;

- ligneux hauts dominés par Acacia raddiana ;

- ligneux bas dominés par Hamada scoparia et Artemisia herba-alba ;

- herbacée pérenne dominée par Stipa tenacissima ;

- chaîne du Jebel Bou Hedma ;

- strates herbacées annuelles ;

- sol nu (zones d'épandage).

Les recouvrements moyens des différentes entités du parc national de Bou Hedma sont représentés dans la figure 9. Le recouvrement moyen des ligneux hauts dominés par Acacia tortilis ne dépasse pas $10 \%$ (photo 6). Une autre entité se localise dans le versant Nord du Jebel Bou Hedma caractérisée par la présence de ligneux bas dominés par Juniperus phenicea et des oléastres qui représentent un taux de recouvrement moyen égal à $7 \%$ (photo 7 ), et un peu dégradée à cause du surpâturage, du défrichement et de l'extension des zones agricoles de la région de Meknassi. 


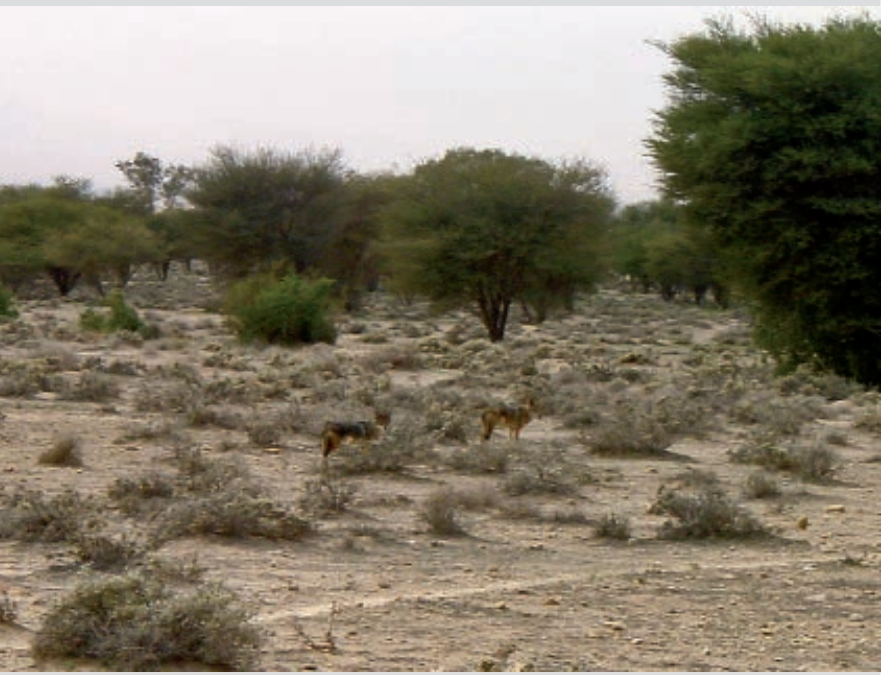

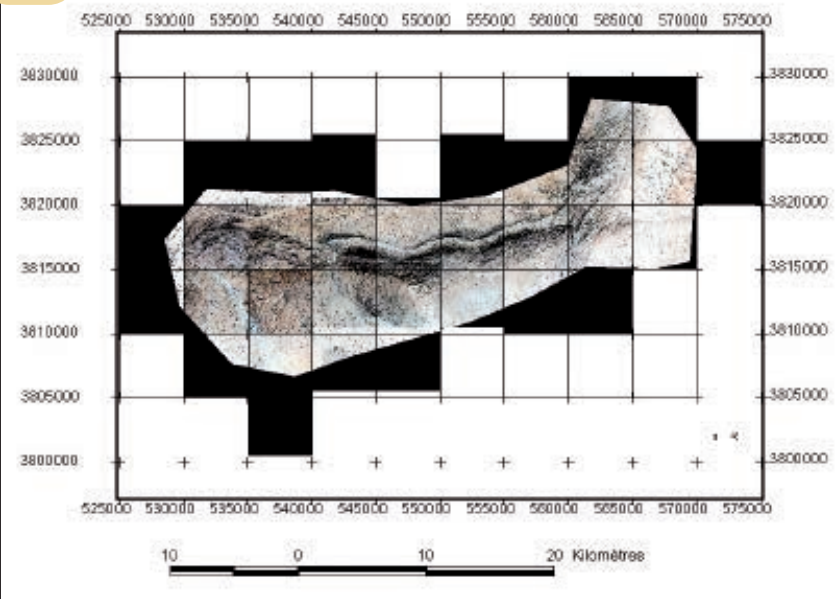

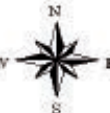

Figure 5.

Mosaïque d'images satellitaires de la région de Bou Hedma (image Quick Bird, septembre 2005).

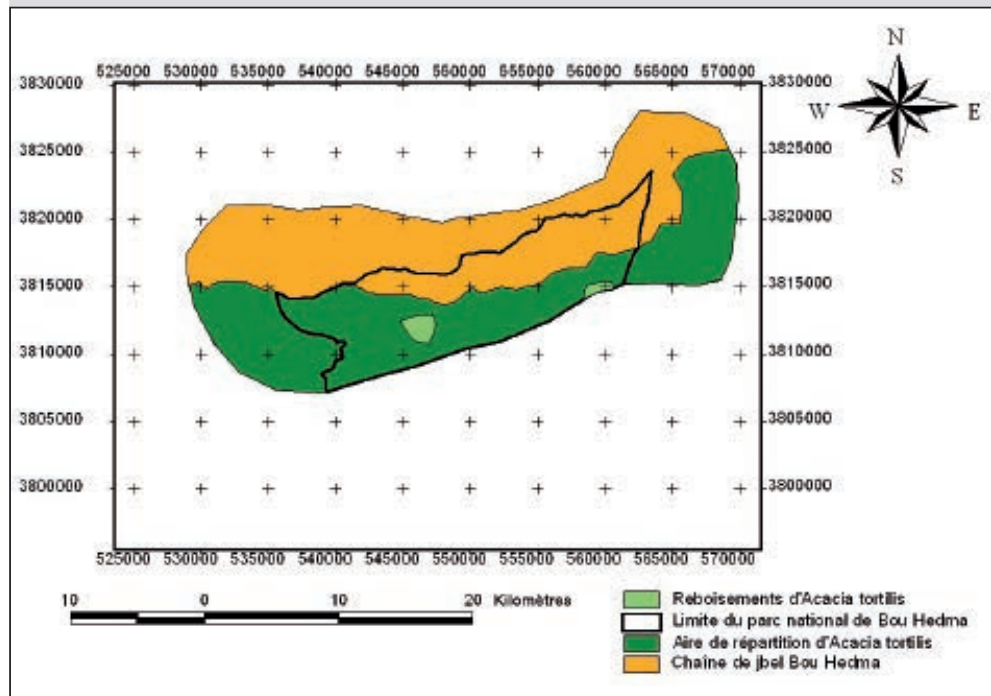

Figure 6.

Aire de la répartition d'Acacia tortilis dans le parc de Bou Hedma et ses alentours (septembre 2005).

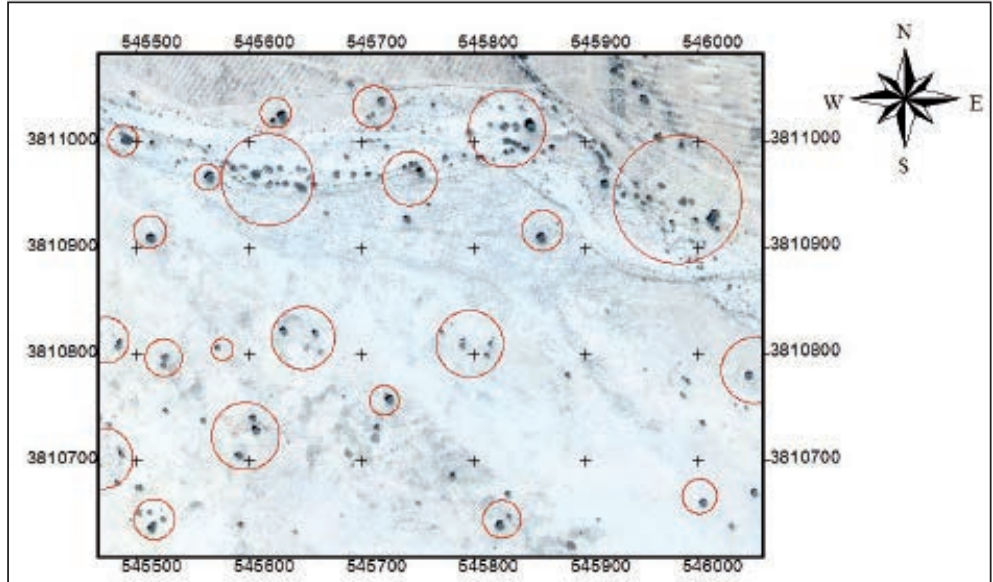

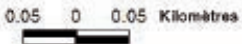

Acacia tortilis

Figure 7.

Répartition des arbres issus de régénération naturelle d'Acacia tortilis dans les zones d'épandage dans le parc de Bou Hedma (Oued Haddej, septembre 2005).
Photo 6.

Peuplement d'Acacia tortilis dans le parc national

de Bou Hedma (2005).

Photo W. Jaouadi.

Les strates herbacées annuelles représentent un recouvrement moyen de $30 \%$ (photo 8). Les aires occupées par Stipa tenacissima ont un recouvrement moyen de l'ordre de $21 \%$ (photo 9), connaissant depuis longtemps le défrichement pour utilisation en artisanat et comme aliment pour le bétail pendant la période de disette. L'entité de ligneux bas dominés par Hamada scoparia et Artemisia herba alba offre un recouvrement moyen de 11 \% (photo 10). Quant à l'entité de ligneux bas dominés par Hamada schmittiana et Atractylis serratuloides, elle présente un taux de recouvrement moyen de $9 \%$.

\section{Élaboration d'une carte de recouvrement d'Acacia tortlis}

La carte de recouvrement (figure 10) d'Acacia tortilis a été établie à partir d'une union spatiale entre la répartition d'Acacia tortilis et la densité de l'espèce dans le parc national de Bou Hedma. La densité est définie comme étant le nombre de cimes par unité de surface (grille de $1 \mathrm{~km}$ de côté). Le recouvrement de l'espèce représente la somme des surfaces de la projection orthogonale des cimes sur le sol. Certaines cimes se chevauchant, la partie chevauchante a été estimée. Les recouvrements ont été divisés par classes afin d'avoir un groupement des placettes de recouvrement presque semblable.

L'analyse de la figure 10 montre que Acacia tortilis présente des densités remarquables dans les reboisements et les plaines, surtout là où subsistent les zones de protection intégrale interdisant tout accès. L'examen du recouvrement de cette espèce indique globalement que le profil « densité » est faible à très faible (10\%) et s'accroît depuis la montagne vers la plaine du parc, notamment en zones d'épandage et dans les lits d'oueds où la disponibilité de l'eau assure une bonne régénération naturelle et le maintien des semis.

La conservation des conditions écologiques de ces peuplements avec de telles densités permet vraisemblablement le maintien d'Acacia tortilis dans cet écosystème steppique. Le recouvrement d'Acacia tortilis reste nettement 


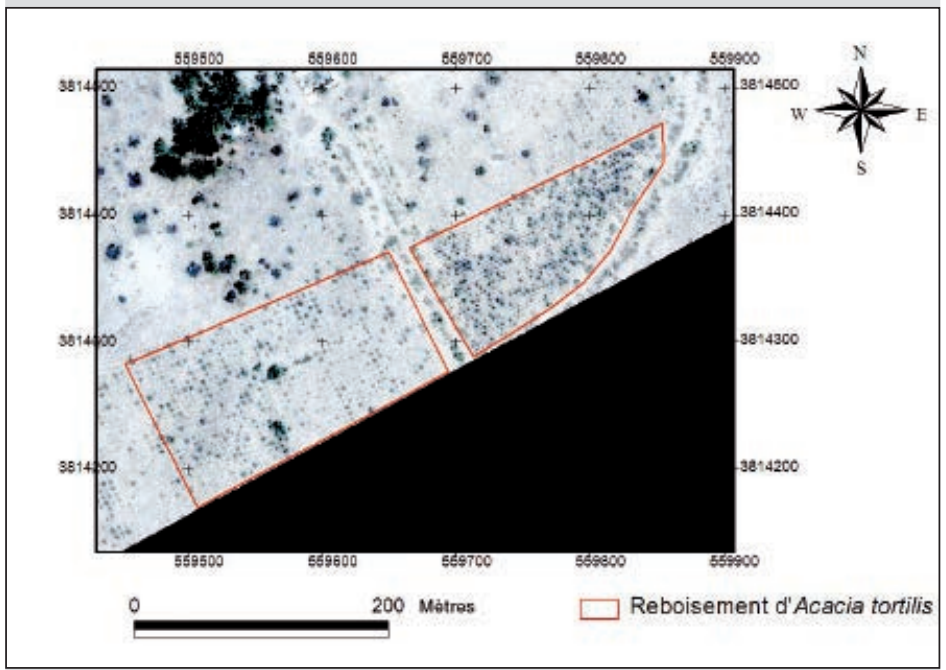

Figure 8.

Parcelle reboisée par Acacia tortilis dans la partie Sud du parc national de Bou Hedma (image satellitaire Quick Bird, septembre 2005).

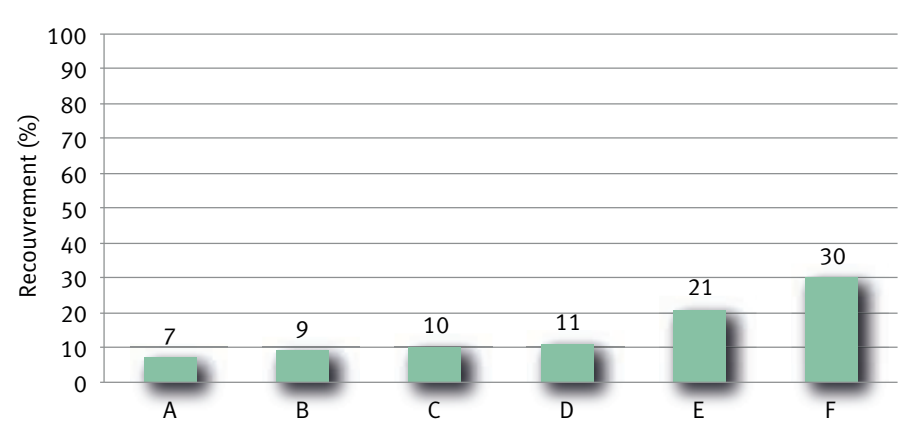

Figure 9.

Recouvrement moyen des différentes entités de végétation du parc national de Bou Hedma (septembre 2005).

A : Ligneux bas dominés par Juniperus phoenicea et Olea europea ; B : Ligneux bas dominés par Hamada schmittia et Atractylis serratuloides ; $C$ : Ligneux hauts dominés par Acacia raddiana; D : Ligneux bas dominés par Hamada scoparia et Artemisia herba-alba ; E : Herbacées pérennes dominées par Stipa tenacissima ; F: Strates herbacées annuelles.

plus important dans les zones de protection intégrale (mises en défens) qu'en dehors de ces zones. Cette densité ne peut que favoriser l'implantation d'une strate herbacée, améliorant ainsi la richesse de la biodiversité des peuplements de cette espèce.

Acacia tortilis se localise à des altitudes variant de 75 à $200 \mathrm{~m}$. La prise en compte de la pente et de l'altitude dans la régénération montre que plus l'altitude et la pente sont fortes, plus la régénération est faible ou absente.

L'effet de la topographie influe amplement sur les conditions météorologiques en favorisant la création de lits d'oued et de zones d'épandage, lieux favorables à la régénération de l'espèce.

Acacia tortilis colonise les sols peu évolués d'apport hydrique, les sols gypseux et les rendzines, avec une préférence pour les sols peu évolués d'apport hydrique qui occupent toute la plaine du versant Sud du Jebel Bou Hedma.

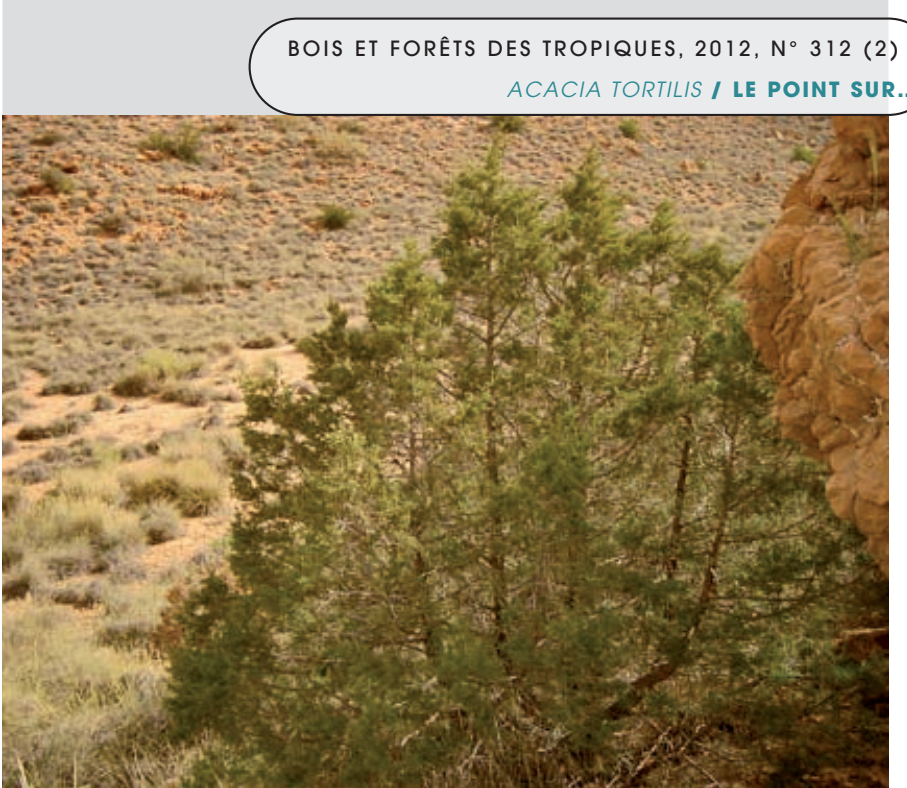

Photo 7.

Juniperus phoenicea sur le versant Nord de Djebel Bou Hedma (2005).

Photo W. Jaouadi.

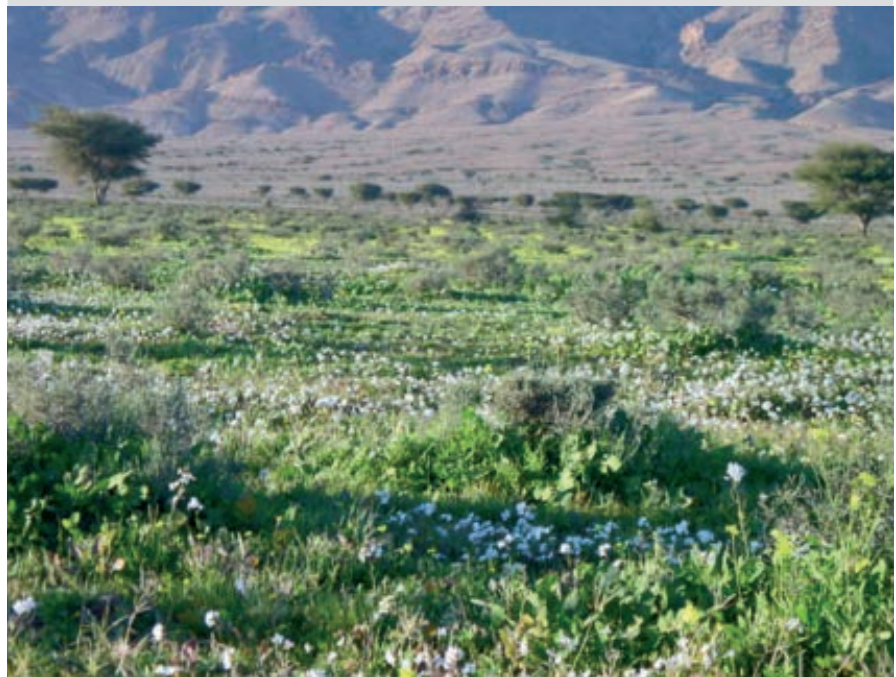

Photo 8.

Strates herbacées annuelles dans la région de Bou Hedma

(2005).

Photo W. Jaouadi.

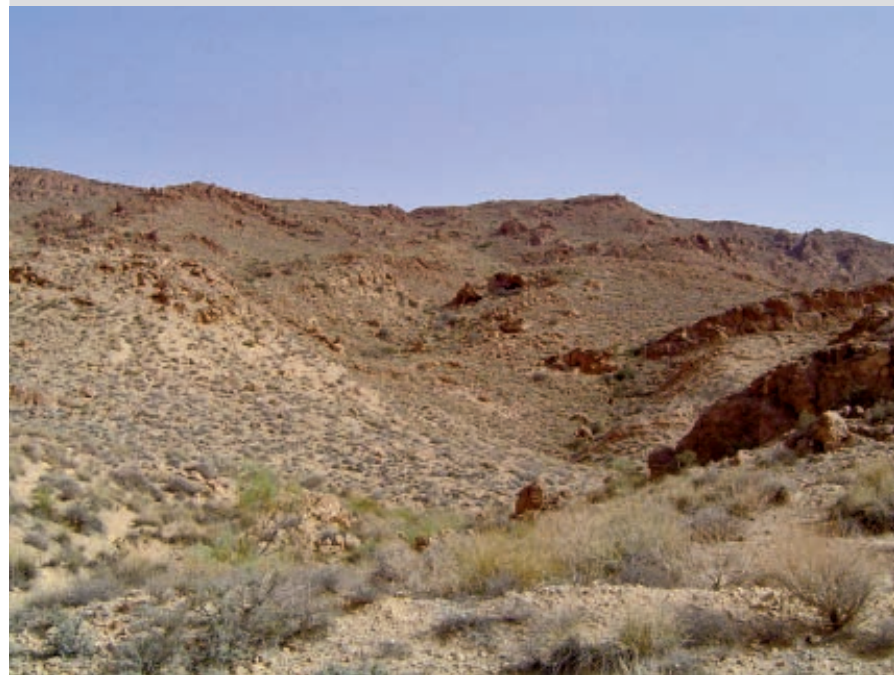

Photo 9.

Glacis de Djebel Bou Hedma dominé par Stipa tenacissima

(2005).

Photo W. Jaouadi. 


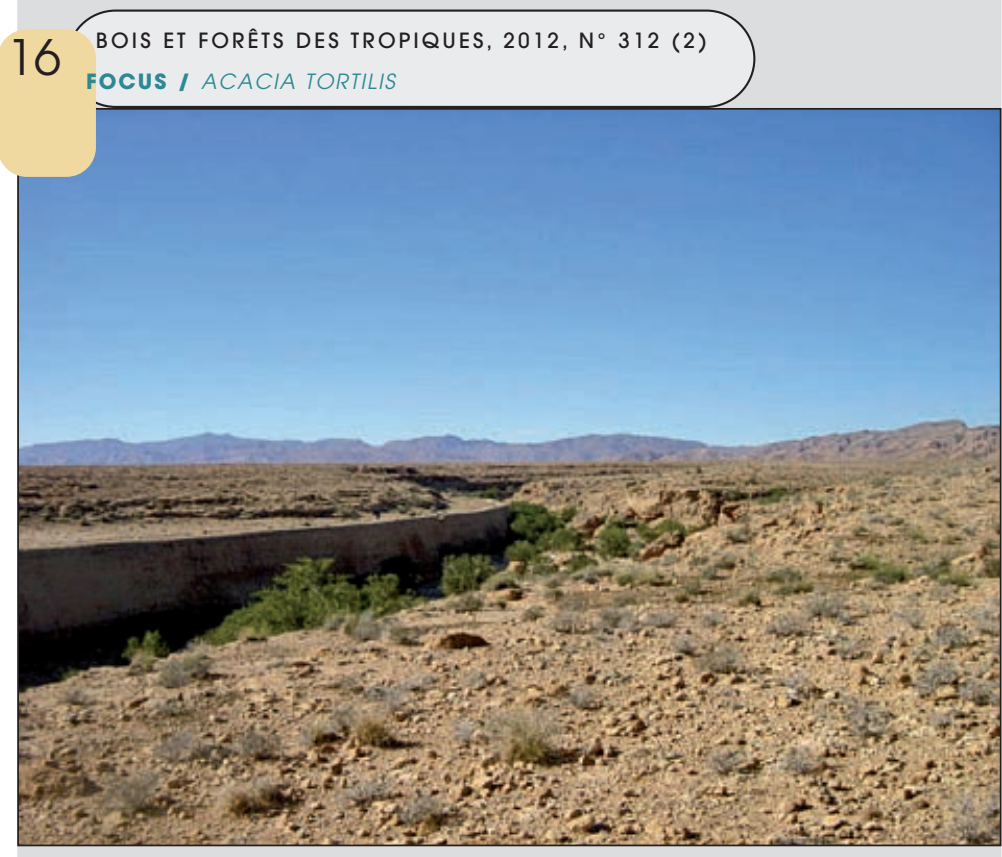

Photo 10.

Entité de ligneux bas dominés par Hamada scoparia et Artemisia herba alba (2005).

Photo W. Jaouadi.

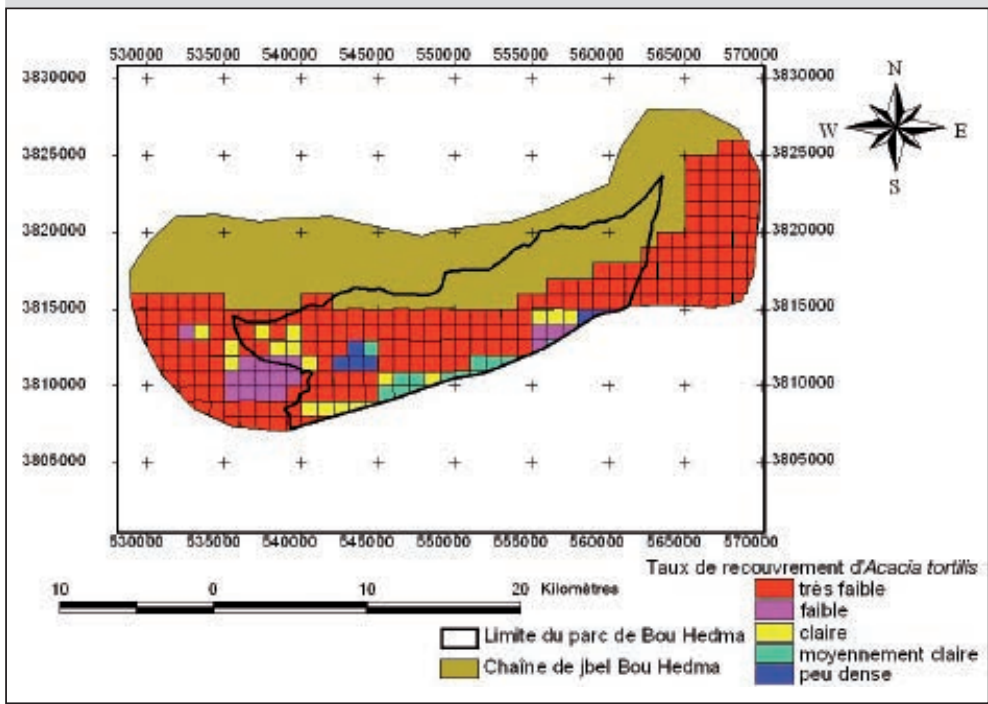

Figure 10.

Recouvrement d'Acacia tortilis dans le parc de Bou Hedma et ses alentours (septembre 2005).

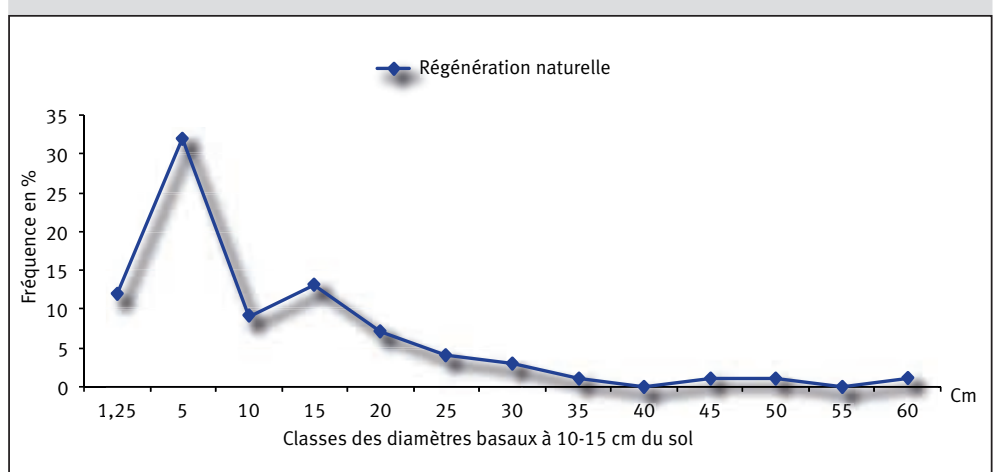

Figure 11.

Structure du peuplement d'Acacia tortilis dans le parc de Bou Hedma.

\section{La structure des peuplements d'Acacia tortilis}

La figure 11 montre l'abondance des individus de diamètres de $5,10,15$ et $20 \mathrm{~cm}$ dans la régénération naturelle. La courbe de fréquence des diamètres présente une allure exponentielle à partir de la classe de diamètre 5 ; ce peuplement est donc de structure jardinée, où tous les âges ou en pratique tous les diamètres sont représentés. Le peuplement peut être jardiné pied par pied ou jardiné par bouquet, aussi la courbe de fréquence des diamètres est-elle représentative d'une fonction sensiblement exponentielle. Cette structure due à la disparition des perturbations externes (anthropique principalement) suite à la protection du parc $\mathrm{a}$ permis le maintien des jeunes semis issus de la régénération naturelle (classe de diamètre inférieure à $10 \mathrm{~cm}$ ) et la protection des autres classes de diamètre contre les coupes illicites. Le manque d'individus de gros diamètre (classe de diamètre supérieure à $35 \mathrm{~cm}$ ) est la conséquence d'une ancienne perturbation (coupe de bois pour la carbonisation et l'utilisation des branches pour des haies et des clôtures).

Pour faire ressortir les différences entre les caractéristiques dendrométriques des peuplements reboisés (P6, P7, $P 8$ et $P 9)$ et ceux dits de régénération naturelle ( $P 1, P 2, P 3$, P4 et P5) d'Acacia tortilis, une analyse comparative a été réalisée (figure 12). D'après cette figure, il ressort que, dans les peuplements naturels, les hauteurs moyennes d'Acacia tortilis varient de $1,66 \mathrm{~m}$ à $3,55 \mathrm{~m}$ contre $0,87 \mathrm{~m}$ à 4,10 m dans les unités de reboisement. Les diamètres moyens des houppiers varient entre $1,81 \mathrm{~m}$ et $4,26 \mathrm{~m}$ dans les peuplements naturels contre $0,67 \mathrm{~m}$ à $5,4 \mathrm{~m}$ dans les parcelles reboisées (figure 13). La figure 14 montre l'évolution des diamètres basaux, qui varient entre $4,98 \mathrm{~cm}$ et $18,71 \mathrm{~cm}$ dans les peuplements naturels, contre $2,5 \mathrm{~cm}$ à $19,95 \mathrm{~cm}$ dans les reboisements.

En se référant aux mesures groupées sur le terrain concernant les mesures dendrométriques des pieds d'Acacia tortilis présents dans les cinq parcelles issues de régénération naturelle, une analyse de variance a été menée, avec dans un premier temps un test de l'égalité des variances à l'aide du test de Hartley. Ce test étant non significatif (rejet de l'égalité des variances), dans un second temps il a été appliqué une transformation angulaire $(Y=$ $2 \operatorname{ArcSin} \sqrt{ } \mathrm{X})$ des caractéristiques dendrométriques dont les valeurs transformées ont été soumises à l'analyse de variance (Anova). L'Anova, représentée dans le tableau I, met en évidence une variation significative entre les parcelles concernant les diamètres basaux et des houppiers des arbres en régénération naturelle. Un effet hautement significatif est également observé pour les hauteurs des arbres. Le classement des parcelles (tableau II) nous permet de constater que la hauteur et le diamètre de houppier varient significativement entre les placettes, faisant apparaître une similitude entre les diamètres moyens des placettes 2, 3 et 4 . D'après les différents classements, les parcelles possèdent des diamètres, hauteurs et diamètres des houppiers moyens variés, caractérisant une régénération naturelle. Les mêmes mesures dendrométriques ont été effectuées également sur des parcelles issues de reboisements réalisés à différentes dates (P6 reboisée en 1963, P7 


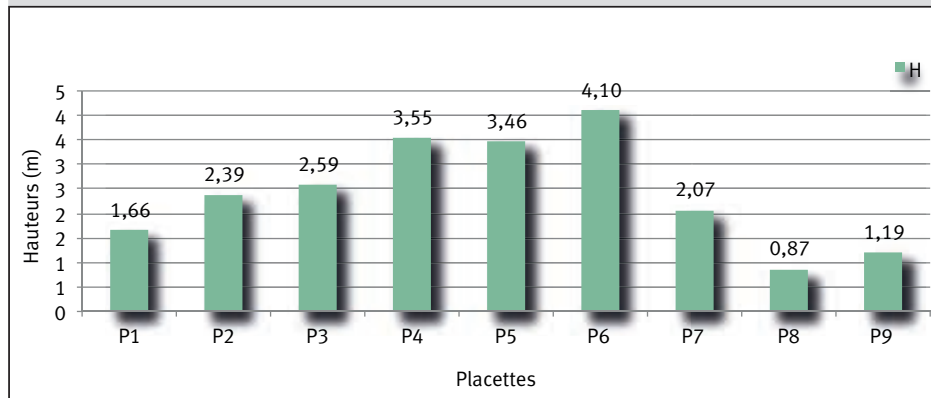

Figure 12.

Variation des hauteurs moyennes $(\mathrm{H})$ en fonction des parcelles échantillonnées.

reboisée en 1967, P8 reboisée en 1994, P9 reboisée en 2000) pour avoir une idée de l'accroissement annuel en diamètre et en hauteur des peuplements d'Acacia tortilis à Bou Hedma. Le tableau III présente les résultats de l'analyse de la variance des accroissements annuels moyens en diamètre et en hauteur des reboisements, dont les accroissements varient significativement en fonction des reboisements. La comparaison des accroissements en diamètre et en hauteur des parcelles issues des reboisements à l'aide du test de Newman et Keuls est représentée dans le tableau IV. Ces analyses révèlent que le reboisement le plus récent de l'année 2000 a les meilleurs accroissements en diamètre $(0,69 \mathrm{~cm} / \mathrm{an})$ et en hauteur $(0,23 \mathrm{~m} / \mathrm{an})$. Ce résultat met en évidence l'effet des travaux mécanisés effectués dans ce reboisement, en comparaison des travaux manuels réalisés dans les reboisements précédents.

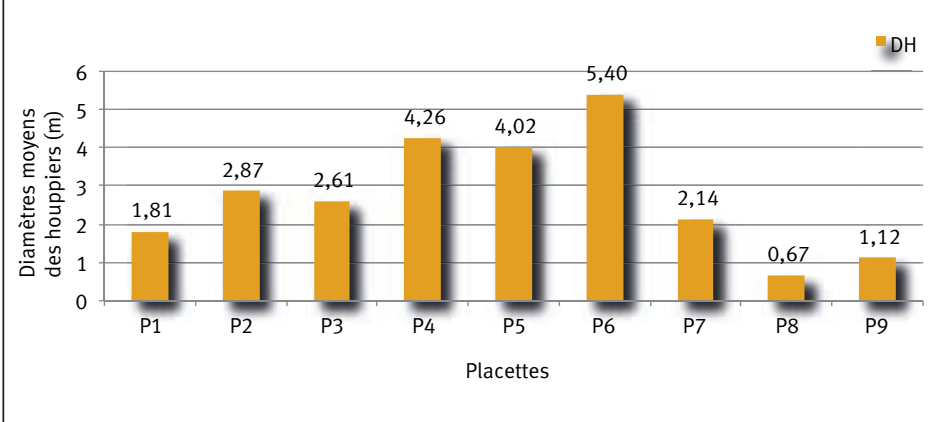

Figure 13.

Variation des diamètres moyens des houppiers (DH) en fonction des parcelles échantillonnées.

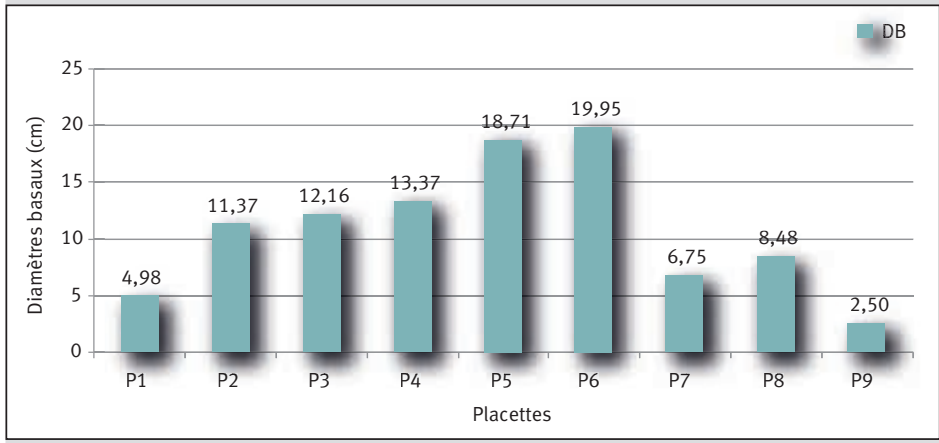

Figure 14.

Variation des diamètres basaux (DB) en fonction des parcelles échantillonnées.

Tableau I.

Analyse de la variance, comparaison des caractéristiques dendrométriques d'Acacia tortilis des cinq parcelles issues de régénération naturelle.

\begin{tabular}{|c|c|c|c|c|c|c|}
\hline Paramètres & $\begin{array}{l}\text { Source de } \\
\text { variation }\end{array}$ & $\begin{array}{l}\text { Degré de } \\
\text { liberté }\end{array}$ & $\begin{array}{l}\text { Somme } \\
\text { des carrés }\end{array}$ & Carré moyen & Valeur de F & $\mathrm{Pr}>\mathrm{F}$ \\
\hline \multirow[t]{3}{*}{ Diamètre basal (cm) } & Placette & 5 & 2379,62 & 594,90 & 4,14 & 0,0037 \\
\hline & Résiduelle & 110 & 15954,91 & 143,73 & & \\
\hline & Totale & 115 & 18334,53 & & & \\
\hline \multirow[t]{3}{*}{ Hauteur (m) } & Placette & 5 & 6,50 & 14,12 & 7,72 & $<0,0001$ \\
\hline & Résiduelle & 110 & 1,83 & & & \\
\hline & Total & 115 & 259,68 & & & \\
\hline \multirow[t]{3}{*}{ Diamètre du houppier (m) } & Placette & 5 & 89,04 & 22,26 & 5,79 & 0,0003 \\
\hline & Résiduelle & 110 & 426,70 & 3,84 & & \\
\hline & Total & 115 & 515,74 & & & \\
\hline
\end{tabular}

F : test d'adéquation à la loi de Fisher.

$\operatorname{Pr}$ : probabilité d'obtenir un résultat aussi extrême si l'hypothèse nulle était vraie (valeur sûre avec $\operatorname{Pr}<0,001$ ).

L'égalité des variances a été testée en recourant au test de Hartley avec $P<0,05$. 
Tableau II.

Comparaison des caractéristiques dendrométriques d'Acacia tortilis des cinq parcelles issues de régénération naturelle à l'aide du test de Newman et Keuls.

\begin{tabular}{l|l|l|l|l|l|l|}
\hline Placette & Dm $(\mathrm{cm})$ & $\begin{array}{l}\text { Similarité } \\
\text { statistique }\end{array}$ & $\mathrm{Hm}(\mathrm{m})$ & $\begin{array}{l}\text { Similarité } \\
\text { statistique }\end{array}$ & Dmh (m) & $\begin{array}{l}\text { Similarité } \\
\text { statistique }\end{array}$ \\
\hline P1 & 4,98 & B & 1,66 & B & & 1,81 \\
\hline P2 & 11,37 & A B & 2,39 & B & 2,87 & A B C \\
\hline P3 & 12,16 & A B & 2,59 & A B & 2,61 & B C \\
\hline P4 & 13,37 & A B & 3,55 & A & 4,26 & A \\
\hline P5 & 18,71 & A & 3,46 & A & 4,02 & A B \\
\hline
\end{tabular}

$\mathrm{Dm}$ : diamètre basal moyen $(\mathrm{cm})$; $\mathrm{Hm}$ : hauteur moyenne $(\mathrm{m})$; Dmh : diamètre moyen du houppier (m).

Tableau III.

Analyse de la variance, comparaison des accroissements annuels en diamètre et en hauteur des peuplements d'Acacia tortilis dans des parcelles issues des quatre dates de reboisement.

\begin{tabular}{|c|c|c|c|c|c|c|}
\hline Paramètres & $\begin{array}{l}\text { Source de } \\
\text { variation }\end{array}$ & $\begin{array}{l}\text { Degré de } \\
\text { liberté }\end{array}$ & $\begin{array}{l}\text { Somme } \\
\text { des carrés }\end{array}$ & $\begin{array}{l}\text { Carré } \\
\text { moyen }\end{array}$ & $\begin{array}{l}\text { Valeur } \\
\text { de F }\end{array}$ & $\mathrm{Pr}>\mathrm{F}$ \\
\hline \multirow[t]{3}{*}{ Accroissement en diamètre (cm/an) } & Placette & 4 & 3,65 & 1,21 & 37,49 & $<0,0001$ \\
\hline & Résiduelle & 126 & 4,12 & 0,03 & & \\
\hline & Totale & 130 & 7,77 & & & \\
\hline \multirow[t]{3}{*}{ Accroissement en hauteur (m/an) } & Placette & 4 & 0,36 & 0,12 & 72,17 & $<0,0001$ \\
\hline & Résiduelle & 126 & 0,21 & 0,001 & & \\
\hline & Totale & 130 & 0,58 & & & \\
\hline
\end{tabular}

Tableau IV.

Comparaison des accroissements moyens annuels en diamètre et en hauteur des parcelles d'Acacia tortilis issues de reboisement réalisé à différentes dates à l'aide du test de Newman-Keuls.

\begin{tabular}{|c|c|c|c|c|}
\hline Placette & $\begin{array}{l}\text { Accroissement en } \\
\text { diamètre (cm/an) }\end{array}$ & $\begin{array}{l}\text { Similarité } \\
\text { statistique }\end{array}$ & $\begin{array}{l}\text { Accroissement en } \\
\text { hauteur (m/an) }\end{array}$ & $\begin{array}{l}\text { Similarité } \\
\text { statistique }\end{array}$ \\
\hline P6 (1963) & 0,46 & $B$ & 0,09 & B \\
\hline P7 (1967) & 0,18 & $\mathrm{C}$ & 0,05 & C \\
\hline P8 (1994) & 0,20 & $C$ & 0,07 & B C \\
\hline P9 (2000) & 0,69 & $A$ & 0,23 & $A$ \\
\hline
\end{tabular}




\section{Discussion}

La superficie de la forêt d'Acacia tortilis en Tunisie ne dépasse guère les 20000 ha, dont 17000 ha constituent le parc national de Bou Hedma (KAREM et al., 1993). Cette forêt risque d'entrer dans la catégorie des écosystèmes en danger si les facteurs défavorables continuent à exercer leurs effets. Selon ZAÂFOURI et al. (2000), la densité à l'hectare de cette forêt a atteint le zéro pied dans la majorité de l'aire de l'espèce et elle est en moyenne de 1 à 10 pieds par hectare dans la zone de Bled Talh. Les résultats montrent que, dans le parc de Bou Hedma, les jeunes sujets de diamètre inférieur à $10 \mathrm{~cm}$ sont dominants, corroborant ceux obtenus par ZAÂFOURI et al. (2000) dans une étude sur la dynamique et l'évolution d'Acacia tortilis dans le parc de Haddej en 1996 et 2000. Toutefois, ces sujets peuvent être considérés comme le résultat d'une bonne régénération naturelle survenue suite à la création de cette zone protégée. Il est possible de conclure que le peuplement d'Acacia tortilis dans le parc de Bou Hedma évolue vers une structure de jeune futaie jardinée, rejoignant ainsi les constats de ZAÂFOURI et al. (1996). Les reboisements mécanisés enregistrent des taux d'accroissement importants en diamètre et en hauteur, par rapport à ceux effectués manuellement. L'étude des images satellitaires permet de vérifier que l'effectif de ces peuplements forestiers est important dans les zones de protection intégrale, favorisé par la régénération naturelle. Dans ce sens, ZNATI (2003) et TARHOUNI (2003) ont montré que la création du parc a permis la préservation et l'augmentation de la superficie d'Acacia tortilis dans les zones de protection intégrale, ainsi qu'une bonne installation de la végétation primitive au sein de cette zone. Cependant, lorsque le nombre des arbres est important dans les plaines en basse altitude, il tend à diminuer d'une manière significative dans les zones en pente et en altitude situées dans le glacis et l'amont de la montagne de Bou Hedma. De même, Mahamadou et al. (2004), travaillant sur la dynamique de la végétation à Haddej Bou Hedma, indiquent qu'il y a des variations significatives du recouvrement végétal et de la richesse floristique. L'examen des images satellitaires a permis d'affirmer que l'espèce colonise fortement les zones d'épandage (oued et ravin). SAHRAOUI et al. (1996) montrent que Acacia tortilis présente des densités remarquables (26 à 50 arbres $/ \mathrm{km}^{2}$ ), là où l'eau s'accumule et serait disponible d'une façon régulière afin d'assurer la croissance des semis ; ces auteurs indiquent aussi que Acacia tortilis, dans les monts de Ougarta (Sahara nord-occidental), s'est régénérée au cours des quarante dernières années surtout dans les zones d'épandage et que son repeuplement est cyclique et suit le rythme de la pluviométrie.

Les supports (photographies aériennes, images satellitaires...) anciens et récents, combinés au système d'information géographique (Sig), sont des outils de travail très importants et fiables pour le suivi et la gestion des écosystèmes forestiers. YVETTE et al. (1998) confirment que ce type d'approche est devenu indispensable pour les sites à large estran, difficiles d'accès, et qui présentent de surcroît une dynamique sédimentaire importante. PANTA et al. (2008) insistent sur le fait que le Sig et la télédétection jouent un rôle majeur dans la production de ces données pour identifier les zones dégradées et déboisées, ainsi que les domaines potentiels pour la conservation. GODERT et al. (2001) confirment que les stratégies de planification pour la gestion dura- ble des terres exigent des données de base solides sur les ressources naturelles (sol, relief, climat, végétation, utilisation des terres, etc.) et sur les aspects socio-économiques, de même que le Sig et la télédétection ont un rôle important dans le couplage et l'analyse de ces données, en particulier pour la détection (directe ou indirecte), l'extrapolation, l'interprétation et le calcul des superficies évoluant à travers le temps. Enfin, l'étude de la végétation au moyen des images satellitaires constitue une démarche efficace permettant une évaluation rapide à travers une cartographie mettant en relief la dynamique de l'occupation des sols et ses répercussions sur les groupements végétaux. L'évaluation correcte de ces changements est fondamentale dans l'élaboration et la mise en place de politiques de protection et de restauration de ces écosystèmes menacés par la pression anthropozoogène et les fluctuations climatiques.

\section{Conclusion}

L'approche spatiale relevés/image couplée aux mesures dendrométriques des peuplements d'Acacia tortilis a permis de cartographier l'aire de distributions de cette espèce dans le parc de Bou Hedma. Une espèce qui se localise essentiellement dans les zones de protection intégrale et dans celles d'épandage. La démarche de mise en protection au sein du parc a permis de s'engager dans la reconstitution de la population de cette espèce et l'augmentation de son effectif. La structure des peuplements d'Acacia tortilis issus de régénération naturelle évolue actuellement vers une structure jardinée caractéristique d'un peuplement forestier naturel suite à l'absence de perturbations anthropiques, à la faveur de la création du parc et de la mise en œuvre de phases de reboisement. L'évaluation correcte de la régénération d'Acacia tortilis est essentielle dans l'élaboration et la mise en place de politiques de protection et de restauration de ces écosystèmes menacés par la pression anthropozoogène et les fluctuations climatiques.

\section{Références bibliographiques}

ANNELISE T., 2000. Application de la télédétection hyperspectrale à la cartographie et l'étude des formations végétales du marais de Kaw. Institut de recherche pour le développement, Centre de Guyane, 180 p.

AUCLAIR L., ZAÂFOURI M. S., 1997. La sédentarisation des nomades dans le sud tunisien : comportements énergétiques et désertification. Sécheresse, 7 (1) : 17-24.

CHAIEB M., BOUKHRIS M., 1998. Flore succincte et illustrée des zones arides et sahariennes de Tunisie. Tunis, Tunisie, L'Or du Temps, $292 \mathrm{p}$.

DAGET P., POISSONET J., 1971. Une méthode d'analyse phytologique des prairies. Critères d'application. Annales d'Agronomie, 22 (1) : 5-41.

DIRECTION GÉNÉRALE DES FORÊTS, 1988. Parcs nationaux de Tunisie. Le Bou Hedma. Tunis, Tunisie, Dgf, 11 p.

FLORET C., PONTANIER R., 1982. L'aridité en Tunisie présaharienne. Paris, France, Orstom, coll. Travaux et documents, $n^{\circ} 150,544 p$. 
GODERT W. J., VAN L., STEPHAN M., 2001. The role of GIS and remote sensing in land degradation assessment and conservation mapping: some user experiences and expectations. International Journal of Applied Earth Observation and Geoinformation, 3 (1): 61-68.

HANAFI A., 2000. Cartographie des systèmes écologiques et étude de leur évolution depuis 1978 dans la région de Menzel Habib (Gabes). Mémoire de diplôme d'études approfondies, Faculté des sciences humaines et sociales de Tunis, Tunisie, $90 \mathrm{p}$.

HENCHI B., 1987. Effet des contraintes hydriques sur l'écologie et l'écophysiologie de Plantago albicans. Thèse de doctorat d'État, Faculté des sciences de Tunis, Tunisie, 360 p.

ISSAFOU M., OUELD BELGACEM A., FERCHIHI A., TARHOUNI M., EL FERJANI E., 2004. Frencing grazing land and GIS, tools for the rangeland long-term management: the observatory of Haddej-Bou Hedma case study. Revue des Régions Arides, Tunisie, 605-610.

JAUFFRET S., 1997. Conditions de la diversité des pelouses oligotrophes à thérophytes (phanérogames et bryophytes) de la plaine des Maures. Mémoire de diplôme d'études approfondies, Université d'Aix-Marseille III, France, 120 p.

JAUFFRET S., 2001. Validation et comparaison de divers indicateurs de changements à long terme dans les écosystèmes méditerranéens arides. Application au suivi de la désertification dans le Sud tunisien. Thèse de doctorat, Université de droit, d'économie et des sciences d'Aix-Marseille, France, $180 \mathrm{p}$.

JENSEN J. R., 1996. Introductory digital image processing: A remote sensing perspective. Upper Saddle River, NJ, ÉtatsUnis, Prentice Hall, $316 \mathrm{p}$.

KAREM A., 2001. Le rôle des parcs nationaux et des réserves naturelles dans la conservation de la biodiversité. Revue des Régions Arides, Tunisie, 242-259.

KAREM A., KSANTINI M., SCHOENENBERGER A., WAIBEL T., 1993. Contribution à la régénération de la végétation dans les parcs nationaux en Tunisie aride. Eschborn, Allemagne, Gtz, 201 p.

LE FLOC'H E., 1973. Étude des parcours du Sud-Tunisien. Carte phytoécologique de Oglat Merteba et Mareth (l/100 000). Annales de l'Institut national de la recherche agronomique de Tunisie, 46, 92 p., 1 carte.

LE HOUÉROU H. N., 1959. Recherches écologiques et floristiques sur la végétation de la Tunisie méridionale. Mémoire, Université d'Alger, Institut de recherches sahariennes, Algérie, 120 p.

LE HOUÉROU H. N., 1969. La végétation de la Tunisie steppique (avec références aux végétations analogues d'Algérie, de Libye et du Maroc). Annales de l'Institut national de la recherche agronomique de Tunisie, 42 (5), $624 \mathrm{p}$.

MAHAMADOU I., OUELD BELGACEM A., FERCHCICHI A., TARHOUNI M., EL FERJANI E., 2004. Fencing grazing land and GIS, tools for the range lands long-term management: the observatory of Haddej-Bouhedma case study. Séminaire international Arido-culture et Cultures Oasiennes. Revue des Régions Arides, pp. 605-610.

NEFFATI M., AKRIMI N., 1996. Études des caractéristiques germinatives des semences de quelques légumineuses spontanées de la Tunisie steppique. Actes de séminaire international. Revue des Régions Arides, Tunisie, 272-287.

PALMER A., FORTESCUE A., 2003. Remote sensing and change detection in rangelands. Vegetatio, 21: 675-680.
PANTA M., KYEHYUN K., CHUDAMANI J., 2008. Temporal mapping of deforestation and forest degradation in Nepal: Applications to forest conservation. Forest Ecology and Management, 256 (9): 1587-1595.

PICOUËT M., SGHAIER M., ZAÂFOURI M. S., 1998. Relation population-environnement en Tunisie désertique. Espace Populations Société, 16 (1) : 53-65.

SAHRAOUI B. S., AIT MOHAND L., ECHAIEB B., 1996. Évolution spatio-temporelle des peuplements d'Acacia tortilis (Forssk.) Hayne raddiana (Savi) Brenan dans les monts Ougarta (Sahara nord-occidental). Sécheresse, 7 : 173-178.

SCHOENENBERGER A., 1986. Premier rapport phyto-écologique sur la flore du parc national de Bou Hedma et l'autoécologie des principales espèces pastorales du Sud. Tunis, Tunisie, Direction générale des forêts, $44 \mathrm{p}$.

SCHOENENBERGER A., 1987. Deuxième rapport phyto-écologique sur les parcs nationaux du Sud, l'amélioration des parcours naturels des zones sahériennes et les espèces algéro-marocaines susceptibles d'être introduites en Tunisie. Tunis, Tunisie, Direction générale des forêts, $26 \mathrm{p}$.

SCHOENENBERGER A., 1988. Troisième rapport phyto-écologique sur les parcs nationaux d'El Feidja, de Bou Hedma, des futurs parcs nationaux de Sidi Toui et Djbil et l'amélioration des parcours naturels des zones arides. Tunis, Tunisie, Direction générale des forêts, $54 \mathrm{p}$.

SCHOENENBERGER A., 1991. Synthèse de l'inventaire floristique des parcs nationaux du Chambi, de Bou Hedma, de Sidi Toui et de Jebil. Tunis, Tunisie, Direction générale des forêts, $48 \mathrm{p}$.

TARHOUNI M., 2003. Cartographie des systèmes écologiques et étude de la dynamique de l'occupation des terres dans le parc national de Bou Hedma. Diplôme d'études approfondies. Faculté des sciences de Tunis, Tunisie, $73 \mathrm{p}$.

TAYEB S., KHÉLOUFI B., 2008. Contribution à l'étude de la dynamique de l'occupation des sols de la plaine de la Macta (Algérie) à l'aide de la télédétection et des systèmes d'information géographique. Comptes Rendus Biologies, 331 (6) : 466-474.

YVETTE M., JEAN-PAUL A., JEAN-PAUL D., 1998. Dynamique sédimentaire de la baie du Mont-Saint-Michel (Ouest France) depuis 1986, à partir de données satellitales. Comptes Rendus de l'Académie des sciences, Séries IIA, Sciences de la terre et des planètes, 327 (3) : 155-159.

ZAÂFOURI M. S., CHAIEB M., 1999. Arbres et arbustes de la Tunisie méridionale menacés de disparition. Acta Botanica Gallica, 146 (4) : 361-373.

ZAÂFOURI M. S., OUELD BELGACEM A., BOUKHRIS M., 2000. Impact de l'anthropisation sur la résistance et la résilience de la forêt d'Acacia tortilis du Bled Talh en Tunisie. Séminaire international. Revue des Régions Arides, Tunisie, 5 p. ZAÂFOURI M. S., ZOUAGHI M., AKRIMI N., JEDDER H., 1996. La forêt steppe à Acacia tortilis subsp. raddiana var. raddiana de la Tunisie aride : dynamique et évolution. In : Séminaire international «Acquis scientifiques et perspectives pour un développement durable des zones arides ", Jerba, Tunisie, 5-7 décembre 1996.

ZNATI S., 2003. Apport de la télédétection et du SIG au suivi de la dynamique de l'occupation du sol en milieu aride tunisien. Cas de l'observatoire Haddej Bou Hedma, Tunisie. Mémoire de master en Sciences et technologie de l'espace, option Télédétection et systèmes d'information géographique, Centre régional africain des sciences et technologies de l'espace en langue française, $85 \mathrm{p}$. 